\title{
Unilateral macular edema in a case of ushers syndrome type 1
}

\author{
Kiran Turaka* \\ Associated Retina Consultants Ltd, Phoenix, USA
}

\begin{abstract}
A 58 year old male with longstanding history of retinitis pigmentosa presented with blurry vision in the right eye since 4 months. Ocular examination revealed retinitis pigmentosa changes in both eyes, epiretinal membrane with macular edema in right eye confirmed by optical coherence tomography and fundus fluorescein angiography. He had bilateral cochlear implants for bilateral hearing loss few years ago.
\end{abstract}

\begin{abstract}
Abbreviations: OD: right eye; OS: left eye; OU: both eyes; ERM: epiretinal membrane; FA: Fluorescein angiography; OCT: optical coherence tomography; RP: retinitis pigmentosa, CME; cystoid macular edema; RPE: retinal pigment epithelium; RP: Retinitis pigmentosa; VA: visual acuity.
\end{abstract}

\section{Case report}

\section{Introduction}

Ushers syndrome (USH) is combination of deafness-blindness disorder. It is characterized by sensorineural hearing loss, retinitis pigmentosa (RP) like changes with vision loss and vestibular changes [1-5]. There are few authors in the medical literature who reported the associated macular edema in patients with Ushers syndrome [3-8]. We hereby report another case of Ushers syndrome type I with unilateral macular edema.

\section{Case description}

A 58-year old Caucasian male with a past diagnosis of retinitis pigmentosa (RP) presented with 4 month history of decreasing in vision in right eye (OD). Visual acuity (VA) was 20/30-2 in OU (both eyes). Anterior segment examination was unremarkable except for sulcus placed posterior chamber intraocular lens. Fundus examination $\mathrm{OU}$ showed vitreous syneresis, pale optic disc with diffuse retinal pigment epithelial (RPE) atrophy, retinal arteriolar attenuation, and bonespicule like pigment in all four quadrants of the retina with an island of pigmented area in the macula. There was an epiretinal membrane (ERM) with retinal thickening in the macula of OD. There were no retinal hemorrhages or exudates in macula OU. Heidelberg-Spectralis optical coherence tomography (OCT) demonstrated the epiretinal membrane (ERM) with cystoid intraretinal retinal edema (Figure 1A) in OD and marked thinning of the retinal pigment epithelium in the left eye (OS) (Figure 1B). Central foveal thickness was 447 microns in OD and 357 microns in OS. Fundus fluorescein angiography (FA) revealed a circular area of hyperfluorescence in the macula OU, with areas of hyperfluorescence in early phases followed by leakage in late phases (Figure 1C) confirming presence of macular edema in OD. There was no evidence of angiographic macular leakage in OS (Figure 1D). $\mathrm{He}$ had bilateral cochlear implant surgery done few years ago for hearing loss. He was diagnosed with Usher's syndrome type I with ERM and cystoid macular edema (CME) in right eye. Treatment options were observation, pars plana vitrectomy with membrane peeling and oral acetazolamide. He was followed by observation. He also was advised to take oral vitamin $\mathrm{A}$ and was informed to avoid intake of excessive vitamin E. However, he will be considered for surgical correction of ERM if there is further decrease in the vision.

\section{Discussion}

Ushers syndrome comprises a group of autosomal recessive inherited disorders. It presents clinically and genetically heterogeneously [1,2]. It is divided into 3 types, of which the most common and severe is type I. Type I patients have profound hearing loss at an early age with RP changes before puberty and problems in maintaining the balance due to difficulty in the vestibular system $[1,2]$. Myosin VIIa (MYO7A) mutations can cause Usher syndrome type I $[4,5]$. Apart from retinitis pigmentosa like changes in the retina, few studies reported associated cystoid macular edema in patients with USH $[3,6-8]$ which was confirmed by OCT and FA. Type II patients have moderate to severe hearing loss and RP changes with onset in late teenage. They usually have a normal vestibular system. Changes in usherin gene (USH2A) are responsible for type II USH. Type III has a variable onset and degree of progressive sensorineural hearing loss and RP $[4,5]$. Currently there is no effective treatment for Ushers syndrome. Cochlear implants will help in patients with hearing loss.

Topical carbonic anhydrase inhibitors are used by various authors to treat CME [6-8]. Genead and his colleague reported in a retrospective study (64 eyes of 32 patients), improvement in visual acuity in $31 \%$ of their patients [6]. They also reported unilateral decrease in central foveal thickness (CFT) in $63 \%$ of the patients and bilateral decrease in CFT in $41 \%$ of the patients [6]. Lemos Reis and associates in a limited prospective interventional case series of patients with USH ( 5 eyes) and RP (23 eyes) with CME utilized topical ketorolac versus dorzolamide [7]. At a follow-up of 1 year, there was statistically significant improvement in VA in patients on ketorolac $(\mathrm{p}=0.02)$ and mild

Correspondence to: Dr. Kiran Turaka, M.D., Associated Retina Consultants Ltd, 1750 E. Glendale, Phoenix AZ 85020, USA, Tel: 215-882-0965; Fax: 215-8820965; E-mail: keen_268@yahoo.com

Key words: ushers syndrome, epiretinal membrane, macular edema, optical coherence tomography, fluorescein angiography

Received: January 20, 2016; Accepted: February 08, 2016; Published: February 12,2016 


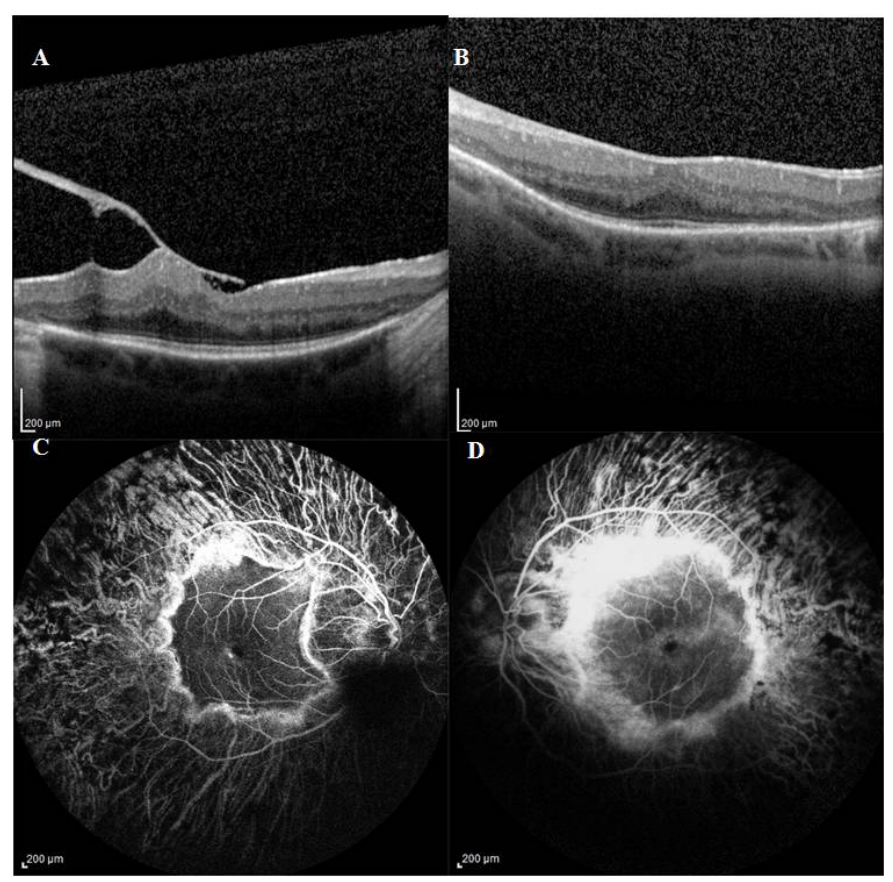

Figure 1. Heidelberg-Spectralis OCT of right eye demonstrating epiretinal membrane with retinal edema due to vitreomacular traction band (1A) and of the left eye showing blunting of the foveal contour with thinning of the RPE (1B). Fundus Fluorescein angiography showing a circular area of hyperfluorescence in the macula of both eyes $(1 \mathrm{C}, \mathrm{D})$ and a pinpoint area of hyperfluorescence inferior to the fovea of the right eye (1C) leading to macular edema and areas of no leakage in the parafoveal region of the left eye (1D). improvement in VA in patients with dorzolamide $(\mathrm{p}=0.20)$. There was decrease in the foveal thickness and foveal zone thickness in patients on ketorolac ( $27 \%$ of eyes) and dorzolamide ( $23 \%$ of eyes) after 1 year of treatment [7]. They concluded that the few selected patients with USH and RP showed improvement in visual acuity with both topical ketorolac and dorzolamide [7]. Our USH patient was considered for observation than oral or topical acetazolamide medication in view of associated ERM that might have lead to the unilateral CME. Future studies are needed in future to report long term visual outcomes of topical anhydrase inhibitors in patients of USH with CME.

\section{References}

1. Keats BJ, Corey DP (1999) The usher syndromes. Am J Med Genet 89: 158-66. [Crossref]

2. Friedman TB, Schultz JM, Ahmed ZM, Tsilou ET, Brewer CC (2011) Usher syndrome: hearing loss with vision loss. Adv Otorhinolaryngol 70: 56-65. [Crossref]

3. Walia S, Fishman GA, Hajali M (2009) Prevalence of cystic macular lesions in patients with Usher II syndrome. Eye (Lond) 23: 1206-1209.[Crossref]

4. Yan D, Liu XZ (2010) Genetics and pathological mechanisms of Usher syndrome. $J$ Hum Genet 55: 327-335.[Crossref]

5. Nájera C, Beneyto M, Millán JM (2005) Usher syndrome: an example of genetic heterogeneity. Med Clin (Barc) 125: 423-427.[Crossref]

6. Genead MA, Fishman GA (2010) Efficacy of sustained topical dorzolamide therapy for cystic macular lesions in patients with retinitis pigmentosa and usher syndrome. Arch Ophthalmol 128: 1146-1150.[Crossref]

7. Lemos Reis RF, Moreira-Gonçalves N, Estrela Silva SE, Brandão EM, Falcão-Reis FM (2015) Comparison of topical dorzolamide and ketorolac treatment for cystoid macular edema in retinitis pigmentosa and Usher's syndrome. Ophthalmologica 233: 43-50. [Crossref]

8. Thobani A, Fishman GA (2011) The use of carbonic anhydrase inhibitors in the retreatment of cystic macular lesions in retinitis pigmentosa and X-linked retinoschisis. Retina 31: 312-315.[Crossref]

Copyright: (C2016 Turaka K. This is an open-access article distributed under the terms of the Creative Commons Attribution License, which permits unrestricted use, distribution, and reproduction in any medium, provided the original author and source are credited. 